\title{
Effect of Allergic Rhinitis and Asthma on Oral Health
}

\author{
Chang-Suk Kim ${ }^{1, \dagger}$ and Kyeong-Soo Lee ${ }^{2}$ \\ 'Department of Dental Hygiene, Ulsan College, Ulsan 44022, \\ ${ }^{2}$ Department of Preventive Medicine and Public Health, Yeungnam University, Daegu 42415, Korea
}

\begin{abstract}
Background: This study aimed to investigate allergic rhinitis and the relationship between allergic diseases, such as asthma and allergic rhinitis, and dental caries and periodontal disease using the raw data from third year of the 6th Korean National Health and Nutrition Examination Survey.

Methods: A total of 3,729 subjects aged over 30 years who underwent examination for allergic diseases and an oral health checkup were selected. The data were analyzed using SPSS IBM SPSS ver. 25.0 (IBM Corp. , USA). Composite sample cross correlation and composite sample logistic regression analyses were performed using the composite sample general linear model.

Results: On examining the socioeconomic characteristics of the subjects suffering from allergic disease and the relationship between allergic disease and oral health, allergic rhinitis was more frequently found in younger subjects with a higher level of education. Periodontal disease and dental caries were more frequent among female, older age groups, lower income earners, and subjects with a lower level of education $(p<0.05)$. On examining the relationship between the oral health characteristics of the subject and allergic diseases and oral health, allergic rhinitis was more common in subjects with a good oral health status perception than those who answered "bad" to the oral health status question. The community periodontal index of treatment needs (CPITN) score was higher in subjects who answered "poor" to the oral health status question, lower frequency of brushing, and higher in subjects using secondary oral hygiene products; the decayed, missing and filled teeth (DMFT) index was higher in subjects with a perception of poor oral health status $(p<0.05)$. The DMFT index was high in the asthma group, and the CPIN score was high in the group who answered "no" to allergic rhinitis.

Conclusion: There is a relationship between asthma and allergic rhinitis and the DFMT index and CPITN score. Corresponding oral programs for allergic patients need to be developed.
\end{abstract}

Key Words: Allergic rhinitis, Asthma, Oral health, Oral hygiene

\section{Introduction}

Allergic rhinitis and asthma are typical allergic diseases ${ }^{1)}$, and their prevalence is increasing every year worldwide ${ }^{2)}$. According to the International Study of Asthma and Allergy in Childhood, the prevalence of the diagnosis of allergic rhinitis has increased to $20.4 \%$ in $2000,28.5 \%$ in 2006 , and $29.9 \%$ in $2010^{3)}$. Allergic rhinitis is associated with bronchial asthma, which is a lower airway inflammatory disease, and develops with a time lag or at the same time ${ }^{4)} .70 \sim 90 \%$ of asthma patients have allergic rhinitis, $20 \sim 50 \%$ of allergic rhinitis patients have asthma $^{5)}$. Allergic diseases are not only caused by genetic factors, but also environmental factors such as change of diet, change in lifestyle and living environment, and increase in air pollution.

Previous studies have shown that beta-2 agonists ( $\beta 2$-agonists), which are used as therapeutic agents in asthmatic patients, affect salivary glands and decrease the salivary secretion and change the salivary components to increase dental caries activity ${ }^{6}$. It induces oral dryness, which causes malocclusion, and results in an oral environment that is susceptible to various oral diseases ${ }^{7)}$. In patients with allergic rhinitis, the typical symptom of 
nasal obstruction increases the total airway resistance and induces oral respiration ${ }^{8)}$, causing oral dryness. These two diseases have commonalities in causing oral and respiratory dryness and have been linked to oral health disorders. In studies using allergic rhinitis as a dependent variable $^{3,8,9)}$ there are no studies on the relevance of oral health.

This study aimed to classify allergic rhinitis, which is the most prevalent among the allergic diseases, and asthma, which is a comorbid disease, as allergic diseases, and to confirm the relationship between periodontal disease and dental caries.

\section{Materials and Methods}

\section{Study population}

This study used raw data from the third year (2015) of the 6th Korean National Health and Nutrition Examination Survey (KNHANES) as research data, and the analysis data were provided after deliberation according to the procedure of using raw data. The subjects were 5,189 adults aged 30 years or older out of the 7,390 who answered a health questionnaire and underwent oral examination. Among these, 3,729 people who did not have any missing values to be included in the analysis were selected as the final study subjects. The health questionnaire of the KNHANES was conducted by the interview method, and the oral examination was conducted by a dentist, based on the World Health Organization criteria.

\section{Research method}

The socioeconomic characteristics of the subjects composed of six items including sex, age, income, educational level, and diagnosis of asthma or allergic rhinitis. The items related to health behavior consisted of smoking, drinking, aerobic exercises, and walking practice. The items related to oral health behavior included lower frequency of brushing, use of auxiliary oral hygiene products, and decayed, missing and filled teeth (DMFT) and community periodontal index of treatment needs (CPITN) indices for oral health. The group with CPITN score of "0" was defined as not having ("No") periodontal disease, and the group with scores " $1 \sim 3$ " as having periodontal disease ("Yes").

\section{Analysis method}

The sample design of the KNHANES was extracted using a two-stage stratified cluster sampling design, and a composite sample planning file was generated to estimate the mean and ratio. The composite sample cross correlation and composite sample logistic regression analyses were performed using the composite sample general linear model. Data analysis was performed using IBM SPSS ver. 25.0 (IBM Corp., Armonk, NY, USA).

\section{Results}

\section{Relationship between socioeconomic characteristics of all subjects, allergic diseases, and oral health}

As a result of examining the socioeconomic characteristics of the subjects and the relationship between allergic disease and oral health, asthma was found to be more frequent in female, older subjects, and those with a lower level of education; allergic rhinitis was more frequent in younger subjects, those with above middle class income, and higher level of education. In the CPITN group, periodontal disease was more frequent in males, older subjects, and those with lower income and lower level of education, while in the DMFT group, dental caries was more frequent in women, older subjects, and those with lower income level and lower level of education (Table 1).

\section{Relevance of allergic disease and oral health according to the health behavior of the subject}

As a result of examining the relationship between the health behavior of the subjects and allergic diseases and oral health, asthma was found more commonly in the in the walking practice group, the group that "does notpractice" group, and allergic rhinitis was more common in the "non-alcoholic" group and in the groups that practiced aerobic exercise. The CPITN score was high in the group that subjectively answered "bad" to subjective general health awareness and in the group that currently smoked, drink, and did not practicing exercise, aerobics, nor 


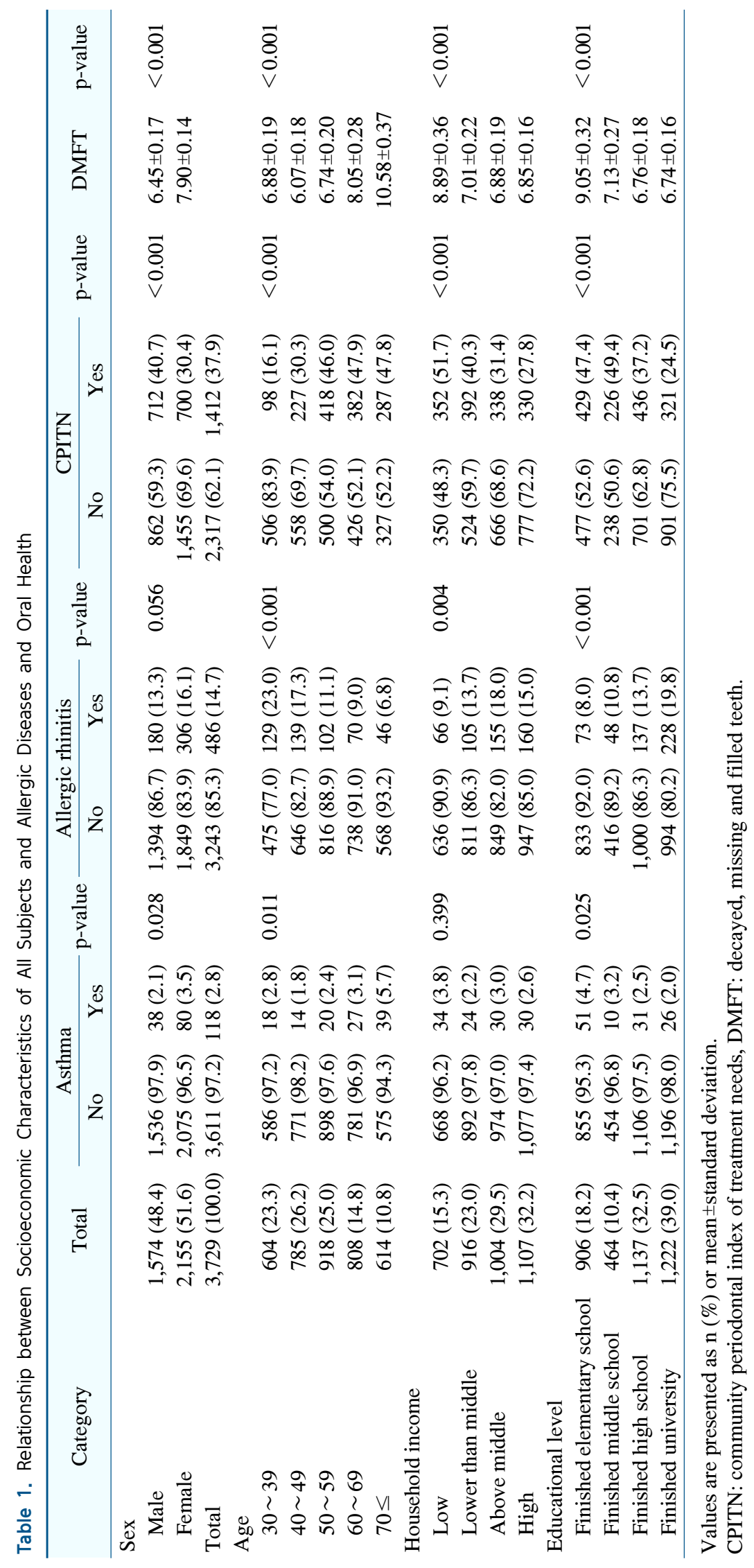




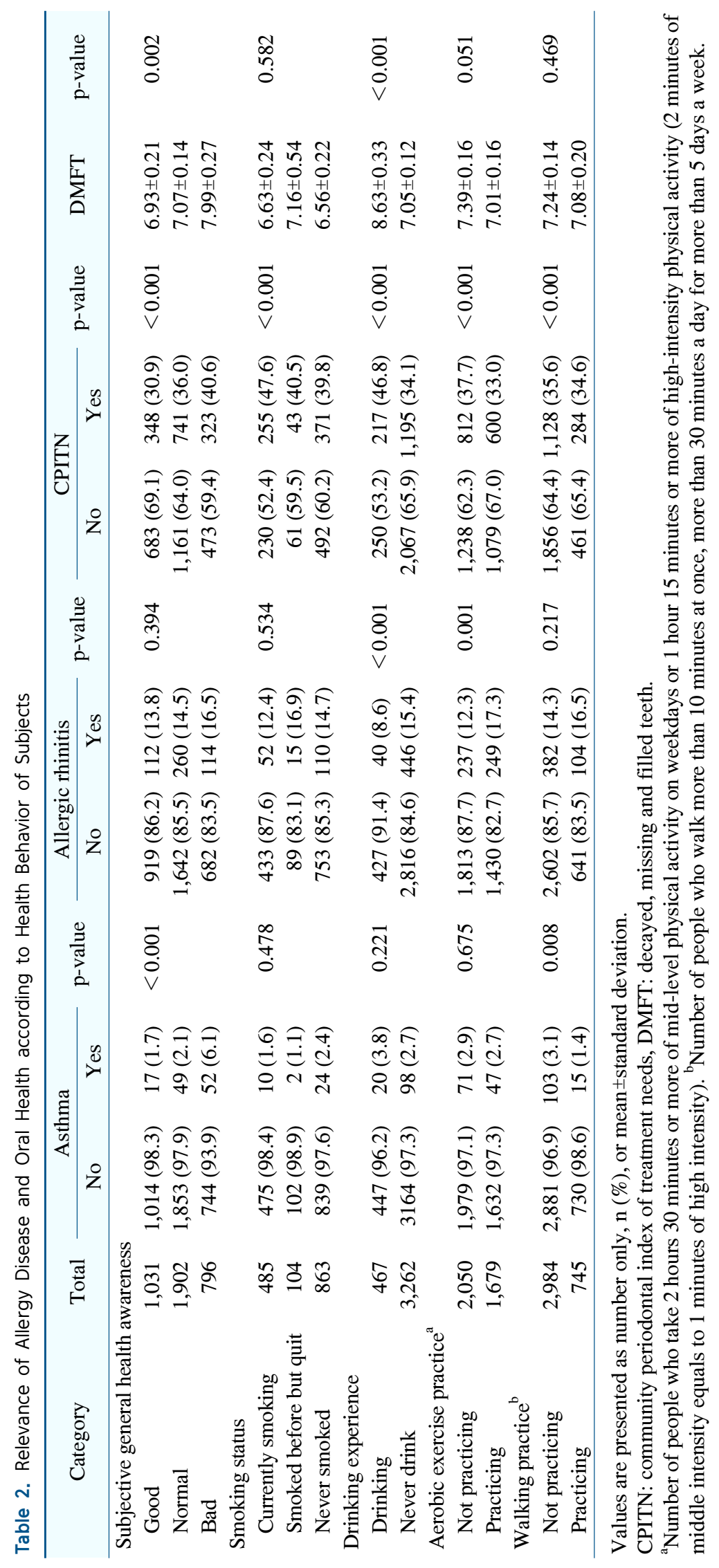




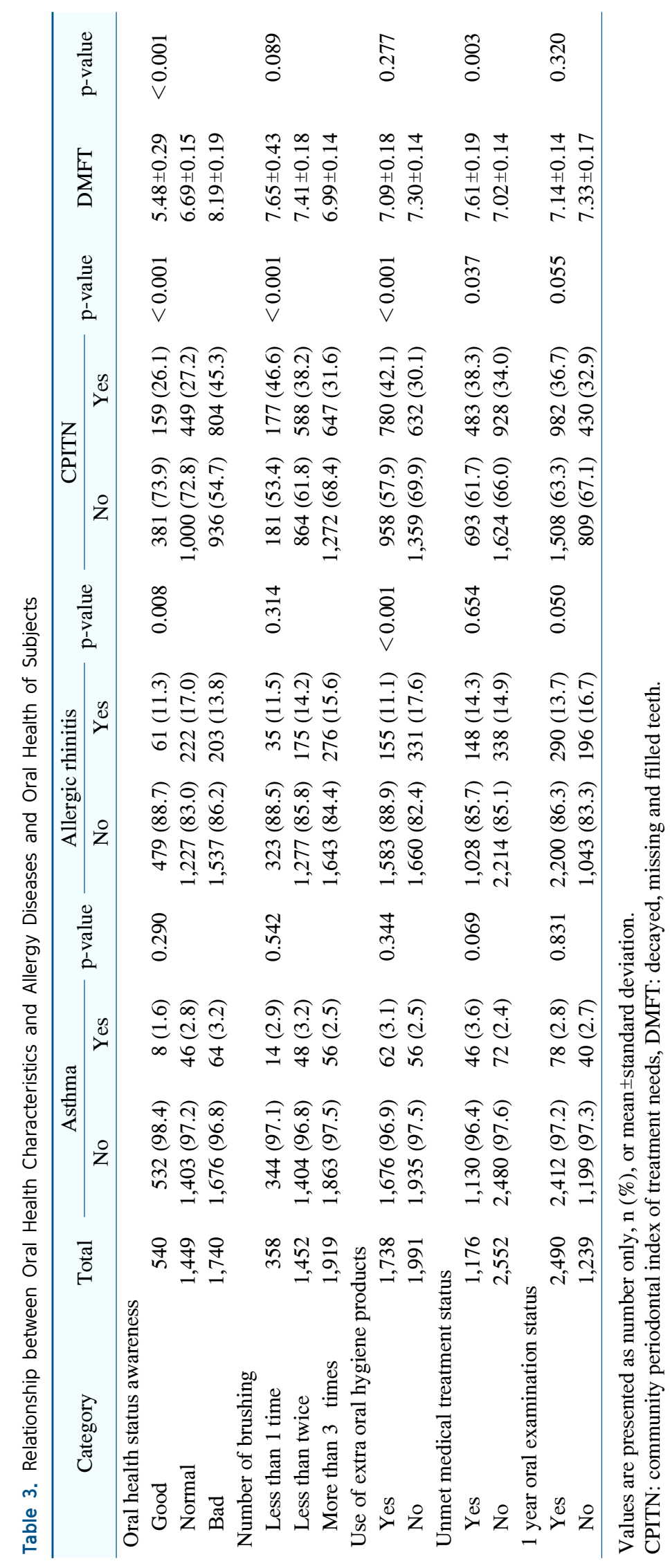


walking. The DMFT index was significantly higher in the group who answered "bad" to subjective general health awareness, and in the group with the habit of alcohol consumption (Table 2).

\section{Relationship between oral health characteristics and allergic diseases and oral health of subjects}

As a result of examining the relationship between the oral health characteristics of the subjects and allergic diseases and oral health, allergic rhinitis was more common in the subjects who answered "bad" to subjective oral health status perception, and in many of the respondents who used oral hygiene products and did not receive a yearly oral examination. The CPITN score was higher in subjects who answered "poor" to subjective oral health status perception, lower frequency of brushing, higher in subjects using secondary oral hygiene products, and the DMFT index was higher in those who considered their oral health status to be poor (Table 3 ).

\section{Relevance of oral health in allergic disease}

Asthma is associated with the DMFT index $(\mathrm{p}<0.05)$. That is, asthma patients had a high DMFT of 8.23.

Allergic rhinitis and CPITN scores were significantly related $(p<0.001)$. That is, $26.5 \%$ of the patients with allergic rhinitis presented with periodontal disease, and $36.9 \%$ of the patients who answered "no" to allergic rhinitis presented with periodontal disease (Table 4).

\section{Logistic regression analysis of factors affecting periodontal disease}

In order to analyze the factors affecting periodontal disease, composite sample logistic regression analysis was performed with statistically significant items as independent variables and periodontal disease as the dependent variable. As a result, it was found that the variables affecting periodontal disease were related to the groups that were older in age, lower in income level, with current smoking habits, and with allergic rhinitis (Table 5).

\section{General linear regression analysis of factors affecting dental caries}

In order to analyze the factors affecting asthma, composite sample general linear regression analysis was performed with statistically significant items as independent variables and asthma as the dependent variable. As a result, it was found that the variables affecting asthma were related to the group that was higher in age and the group that subjectively opined that their oral health is bad (Table 6).

\section{Discussion}

The purpose of this study was to evaluate the relationship between allergic rhinitis and asthma, and periodontal disease and dental caries using the 2015 KNHANES.

Results of the study showed that allergic rhinitis and asthma were more prevalent in female, and allergic rhinitis was more prevalent in the younger subjects, while asthma in the older subjects. The results were in line with the research reported by Chala et al. ${ }^{10)}$. On the contrary, Song and $\mathrm{Cho}^{11)}$ found a higher number of male in a study on

Table 4. Relevance of Oral Health to Allergic Disease

\begin{tabular}{|c|c|c|c|c|c|c|}
\hline \multirow{2}{*}{ Category } & \multirow{2}{*}{ Total } & \multicolumn{2}{|c|}{ CPITN } & \multirow{2}{*}{$\mathrm{p}$-value } & \multirow{2}{*}{ DMFT } & \multirow{2}{*}{ p-value } \\
\hline & & No & Yes & & & \\
\hline \multicolumn{7}{|l|}{ Asthma } \\
\hline No & 3,611 & $2,242(64.6)$ & $1,369(35.4)$ & 0.842 & $7.18 \pm 0.12$ & 0.028 \\
\hline Yes & 118 & $75(65.7)$ & $43(34.3)$ & & $8.23 \pm 0.60$ & \\
\hline \multicolumn{7}{|c|}{ Allergic rhinitis } \\
\hline No & 3,243 & $1,967(63.1)$ & $1,276(36.9)$ & $<0.001$ & $7.26 \pm 0.13$ & 0.138 \\
\hline Yes & 486 & $350(73.5)$ & $136(26.5)$ & & $6.88 \pm 0.26$ & \\
\hline
\end{tabular}

Values are presented as number only, $\mathrm{n}(\%)$, or mean \pm standard deviation.

CPITN: community periodontal index of treatment needs, DMFT: decayed, missing and filled teeth. 
Table 5. Logistic Regression Analysis of Factors Affecting Periodontal Disease

\begin{tabular}{|c|c|c|c|c|c|c|}
\hline Category & $\mathrm{B}$ & SE & $95 \% \mathrm{CI}$ & $\mathrm{t}$ & p-value & Odds ratio \\
\hline \multicolumn{7}{|l|}{ Sex (ref. female) } \\
\hline Male & 0.294 & 0.207 & $(-0.115 \sim 0.702)$ & 1.421 & 0.157 & 1.342 \\
\hline \multicolumn{7}{|l|}{ Age (ref. 30 years old) } \\
\hline $40 \sim 49$ & 0.869 & 0.241 & $(0.393 \sim 1.344)$ & 3.611 & $<0.001$ & 2.383 \\
\hline $50 \sim 59$ & 1.454 & 0.257 & $(0.946 \sim 1.961)$ & 5.656 & $<0.001$ & 4.278 \\
\hline $60 \sim 69$ & 1.484 & 0.254 & $(0.983 \sim 1.985)$ & 5.852 & $<0.001$ & 4.410 \\
\hline$\geq 70$ & 1.353 & 0.281 & $(0.798 \sim 1.908)$ & 4.818 & $<0.001$ & 3.869 \\
\hline \multicolumn{7}{|l|}{ Household income (ref. high) } \\
\hline Low & 0.701 & 0.237 & $(0.233 \sim 1.170)$ & 2.955 & 0.004 & 2.016 \\
\hline Lower than middle & 0.479 & 0.224 & $(0.036 \sim 0.922)$ & 2.137 & 0.034 & 1.615 \\
\hline Above middle & 0.149 & 0.207 & $(-0.260 \sim 0.557)$ & 0.720 & 0.472 & 1.161 \\
\hline \multicolumn{7}{|c|}{ Educational level (ref. university graduate or more) } \\
\hline Finished elementary school & -0.073 & 0.251 & $(-0.569 \sim 0.423)$ & -0.291 & 0.772 & 0.930 \\
\hline Finished middle school & 0.426 & 0.217 & $(-0.003 \sim 0.854)$ & 1.963 & 0.051 & 1.531 \\
\hline Finished high school & 0.164 & 0.177 & $(-0.186 \sim 0.514)$ & 0.925 & 0.356 & 1.178 \\
\hline \multicolumn{7}{|c|}{ Smoking status (ref. non-smoking) } \\
\hline Currently smoking & 0.585 & 0.148 & $(0.292 \sim 0.878)$ & 3.943 & $<0.001$ & 1.794 \\
\hline Smoked before but quit & 0.412 & 0.259 & $(-0.100 \sim 0.924)$ & 1.588 & 0.114 & 1.510 \\
\hline \multicolumn{7}{|c|}{ Drinking status (ref. not drinking) } \\
\hline Drinking currently & -0.218 & 0.360 & $(-0.930 \sim 0.493)$ & -0.606 & 0.545 & 0.804 \\
\hline \multicolumn{7}{|c|}{ Number of brush (ref. more than 3 times) } \\
\hline 2 times & 0.090 & 0.208 & $(-0.320 \sim 0.500)$ & 0.433 & 0.665 & 1.094 \\
\hline Less than 1 time & -0.050 & 0.141 & $(-0.329 \sim 0.228)$ & -0.358 & 0.721 & 0.951 \\
\hline \multicolumn{7}{|c|}{ Use oral hygiene products (ref. do not use) } \\
\hline Use & 0.024 & 0.130 & $(-0.233 \sim 0.281)$ & 0.183 & 0.855 & 1.024 \\
\hline \multicolumn{7}{|l|}{ Allergic rhinitis (ref. yes) } \\
\hline No & 0.318 & 0.187 & $(-0.051 \sim 0.686)$ & 1.702 & 0.019 & 1.374 \\
\hline Constant term & -2.218 & 0.469 & $(-3.145 \sim-1.292)$ & -4.728 & $<0.001$ & \\
\hline
\end{tabular}

SE: standard error, CI: confidence inteval.

domestic elderly asthma patients. In a cohort study including elderly asthma patients. Suggested that about $90 \%$ of the patients developed the disease beyond the age of 40 years, and about $50 \%$ of the patients developed it beyond the age of 60 years. Hence, pediatric asthma symptoms were different from those of the older subjects. Greiner et al. ${ }^{12)}$ suggested that allergic rhinitis is a common disease affecting all age groups, which can be a source of many complications that could affect their quality of life. The prevalence of allergic rhinitis was higher with higher income and education levels, and that of asthma was higher with a lower level of education. Although allergic diseases are highly related to demographic sociological factors, most domestic studies related to allergic rhinitis are aimed at children. Existing studies on asthma also do not include overall age and demographic factors. The increasing prevalence of asthma in Korea, the aging population, lifestyle changes, and the worsening of air pollution will increase the number of patients with allergic diseases. The CPITN scored and DMFT index were noted to be higher with age and lower household income and education level, which is consistent with the results of a study by Shin et al. ${ }^{13)}$. As age increases, the prevalence of alveolar bone increases with an increase in plaque or calculus, which in turn increases the prevalence of periodontal disease and poor oral environment due to the lack of nutritional intake and systemic diseases. As a result of the lack of preventive consciousness for oral disease and the burden of treatment costs, the treatment time is thought to be missed, but further research is needed to provide more clarity of evidence.

As for health behavior, the prevalence of asthma was 
Table 6. General Linear Regression Analysis of Factors Affecting Dental Caries

\begin{tabular}{|c|c|c|c|c|c|}
\hline Category & $\mathrm{B}$ & SE & $95 \% \mathrm{CI}$ & $\mathrm{t}$ & p-value \\
\hline \multicolumn{6}{|l|}{ Sex (ref. male) } \\
\hline Female & -1.380 & 0.196 & $(-1.768 \sim-0.992)$ & -7.023 & $<0.001$ \\
\hline \multicolumn{6}{|l|}{ Age (ref. 30 years old) } \\
\hline $40 \sim 49$ & -0.726 & 0.257 & $(-1.233 \sim-0.219)$ & -2.828 & 0.005 \\
\hline $50 \sim 59$ & -0.129 & 0.296 & $(-0.713 \sim 0.455)$ & -0.435 & 0.664 \\
\hline $60 \sim 69$ & 1.147 & 0.367 & $(0.422 \sim 1.872)$ & 3.125 & 0.002 \\
\hline$\geq 70$ & 3.380 & 0.457 & $(2.477 \sim 4.283)$ & 7.392 & $<0.001$ \\
\hline \multicolumn{6}{|l|}{ Household income (ref. high) } \\
\hline Low & 0.404 & 0.362 & $(-0.311 \sim 1.120)$ & 1.117 & 0.266 \\
\hline Lower than middle & -0.324 & 0.244 & $(-0.805 \sim 0.158)$ & -1.328 & 0.186 \\
\hline Above middle & -0.165 & 0.223 & $(-0.605 \sim 0.275)$ & -0.742 & 0.459 \\
\hline \multicolumn{6}{|c|}{ Education level (ref. university graduate or more) } \\
\hline Finished elementary school & -0.073 & 0.402 & $(-0.867 \sim 0.721)$ & -0.182 & 0.856 \\
\hline Finished middle school & -0.667 & 0.320 & $(-1.298 \sim-0.036)$ & -2.087 & 0.038 \\
\hline Finished high school & -0.293 & 0.225 & $(-0.738 \sim 0.151)$ & -1.303 & 0.194 \\
\hline \multicolumn{6}{|c|}{ Drinking status (ref. not drinking) } \\
\hline Drinking currently & 0.072 & 0.310 & $(-0.540 \sim 0.684)$ & 0.231 & 0.817 \\
\hline \multicolumn{6}{|c|}{ Subjective general health awareness (ref. good) } \\
\hline $\mathrm{Bad}$ & -0.362 & 0.323 & $(-1.000 \sim 0.277)$ & -1.118 & 0.265 \\
\hline Normal & -0.428 & 0.230 & $(-0.882 \sim 0.027)$ & -1.859 & 0.065 \\
\hline \multicolumn{6}{|c|}{ Oral health status awareness (ref. good) } \\
\hline $\mathrm{Bad}$ & 2.818 & 0.309 & $(2.208 \sim 3.428)$ & 9.121 & $<0.001$ \\
\hline Normal & 1.482 & 0.271 & $(0.946 \sim 2.018)$ & 5.460 & $<0.001$ \\
\hline \multicolumn{6}{|l|}{ Diagnosis of asthma (ref. yes) } \\
\hline No & 0.225 & 0.573 & $(-0.907 \sim 1.357)$ & 0.392 & 0.695 \\
\hline Constant term & 6.136 & 0.481 & $(5.186 \sim 7.086)$ & 12.755 & $<0.001$ \\
\hline
\end{tabular}

SE: standard error, CI: confidence inteval.

higher in the group without the habit of exercise, and the prevalence of allergic rhinitis was higher in the group without any drinking experience. It is difficult to explain the causal relationship between "cannot practice walking" due to asthma and "asthma occurred due to absence of walking practice"; hence, further research is needed in this area. The CPITN score was higher in the group that perceived subjective systemic health awareness as "bad," and higher in the group who smoked, had a drinking habit, and did not exercise. The study by $\mathrm{Kim}$ and Choi ${ }^{14)}$ supports the results of this study because the practice of exercise and periodontal disease are related, and smoking and drinking have a direct effect on periodontal disease. The DMFT index was higher in the group that perceived their subjective overall health awareness as "bad" and in subjects with a drinking habit.

Regarding oral health behaviors, fewer the number of brush strokes, higher the prevalence of periodontal disease and dental caries. If frequency of brushing or the habit of practicing auxiliary oral hygiene is low, the oral environment deteriorates, and there is an increase in oral diseases as a natural result. According to the 2010 National Health Survey Report, the use of dental floss was limited to $14.65 \%$ of the population, indicating that the need for dental flossing and interdental toothbrush training is urgently needed. In addition, the DMFT index was high in the subjects who had not undergone oral examination for one year.

The association between asthma and dental caries was interpreted using average DMFT index. The score was 8.23 in asthmatic patients, and this was significantly higher than that of non-asthmatic subjects $(7.18 ; \mathrm{p}<0.05)$; these results were consistent with the findings of Choi ${ }^{15}$ and Alavaikko et al. ${ }^{16)}$. In Choi's study ${ }^{15)}$, the average DMFT index for asthma patients was 7.92, which is lower than the results of this study. However, the relationship 
between the two diseases was thereby confirmed. Alavaikko et al. ${ }^{16)}$ reported of doubling of the risk of dental caries in asthma patients, and doctors and dentists emphasized the need to develop dental caries prevention programs for asthma patients. Bozejac et al. ${ }^{17)}$ reported that asthma patients had no oral health education, and Ryberg et al. ${ }^{18)}$ suggested that asthma patients have increased susceptibility to dental caries but poor oral hygiene management. In order to prevent poor oral health in asthma patients, it is necessary to educate them regarding the importance of oral health, aid them with appropriate oral health management, and develop programs to prevent oral diseases.

There was an inverse relationship between allergic rhinitis and periodontal disease $(\mathrm{p}<0.001)$. Previous studies like those by Friedrich et al. ${ }^{19)}$ showed an inverse association of allergic disease with periodontitis, and these are consistent with the results of this study. These results were in contrast to the hypothesis at the beginning of the study, but many allergic diseases and otolaryngologyrelated studies are questioning the relationship between allergic rhinitis and periodontal disease. It is significant that no similar studies have been attempted in Korea until now. Biological mechanisms of hygiene hypotheses about how periodontal pathogens affect allergic rhinitis ${ }^{20,21)}$ or the oral breathing habit due to nasal congestion in the allergic rhinitis patients are thought to adversely affect oral disease. However, to provide more solid evidence, this study suggests the need for follow-up studies that add variables such as lifestyle and drug use in allergic rhinitis patients.

The limitation of the study is that it may be difficult to explain the causal relationship due to the limitations of cross-sectional examination. A prospective cohort study may be necessary because the CPITN test is limited to representative tooth use, and the prevalence of periodontitis may be overestimated or underestimated. Nevertheless, by using the large-scale data from the KNHANES a representative data of the Korean population, the results were meaningful because domestic studies confirmed the relationship between allergic diseases and oral health. Allergic diseases include allergic rhinitis, asthma, and atopic dermatitis. All three diseases were analyzed in this study; as atopic dermatitis was not related to oral health, it was excluded from the final results.

In the future, the development of oral intervention programs reflecting the characteristics of oral health of allergy patients and the development of dental hygiene specialists who can be dedicated to the education and provision of oral health care in allergy patients will be necessary.

\section{Notes}

\section{Conflict of interest}

No potential conflict of interest relevant to this article was reported.

\section{Ethical approval}

The KNHANES was approved by the KCDC Institutional Review Board (2015-01-02-6C).

\section{ORCID}

Chang-Suk Kim, https://orcid.org/0000-0001-6991-5338

Kyeong-Soo Lee, https://orcid.org/0000-0001-8183-9462

\section{Acknowledgements}

This work was supported by the 2018 Research Fund of Ulsan College.

\section{References}

1. Brozek JL, Bousquet J, Baena-Cagnani CE, et al.: Allergic Rhinitis and its Impact on Asthma (ARIA) guidelines: 2010 revision. J Allergy Clin Immunol 126: 466-476, 2010. https://doi.org/10.1016/j.jaci.2010.06.047

2. Worldwide variation in prevalence of symptoms of asthma, allergic rhinoconjunctivitis, and atopic eczema: ISAAC. The International Study of Asthma and Allergies in Childhood (ISAAC) Steering Committee. Lancet 351: 1225-1232, 1998. https://doi.org/10.1016/s0140-6736(97)07302-9

3. Hong SJ, Ahn KM, Lee SY, Kim KE: The prevalences of asthma and allergic diseases in Korean children. Korean $\mathbf{J}$ Pediatr 51: 345-350, 2008.

4. Westman M, Stjärne P, Asarnoj A, et al.: Natural course and comorbidities of allergic and nonallergic rhinitis in children. $\mathbf{J}$ 
Allergy Clin Immunol 129: 403-408, 2012.

https://doi.org/10.1016/j.jaci.2011.09.036

5. Leynaert B, Neukirch F, Demoly P, Bousquet J: Epidemiologic evidence for asthma and rhinitis comorbidity. J Allergy Clin Immunol 106(5 Suppl): S201-S205, 2000. https://doi.org/10.1067/mai.2000.110151

6. Mazzoleni S, Stellini E, Cavaleri E, Angelova Volponi A, Ferro R, Fochesato Colombani S: Dental caries in children with asthma undergoing treatment with short-acting beta2-agonists. Eur J Paediatr Dent 9: 132-138, 2008.

7. Tanaka LS, Dezan CC, Parron Fernandes KB, et al.: The influence of asthma onset and severity on malocclusion prevalence in children and adolescents. Dental Press J Orthod 17: 50-57, 2012. http://doi.org/10.1590/S2176-94512012000100007

8. Cho SH: Recent update of local allergic rhinitis. Allergy Asthma Respir Dis 1: 303-308, 2013. https://doi.org/10.4168/aard.2013.1.4.303

9. Lee KS, Rha YH: Research on pediatric allergic rhinitis in Korea. Allergy Asthma Respir Dis 6: 58-65, 2018. https://doi.org/10.4168/aard.2018.6.s1.s58

10. Chala S, Rouiffi S, Soualhi M, Bourkadi JE, Abouqal R, Abdallaoui F: Association between untreated carious lesions and asthma in adults at Rabat University Hospital, Morocco: a cross sectional study. BMC Res Notes 10: 221, 2017. https://doi.org/10.1186/s13104-017-2548-2

11. Song WJ, Cho SH: Challenges in the management of asthma in the elderly. Allergy Asthma Immunol Res 7: 431-439, 2015. https://doi.org/10.4168/aair.2015.7.5.431

12. Greiner AN, Hellings PW, Rotiroti G, Scadding GK: Allergic rhinitis. Lancet 378: 2112-2122, 2011. https://doi.org/10.1016/S0140-6736(11)60130-X

13. Shin MS, Hwang MY, Kim SK: Relationship of oral health awareness to oral health indexes among adults. J Dent Hyg Sci 12: 607-616, 2012.

14. Kim CS, Choi YK: Survey of adults' perceptions of the association between chronic diseases and oral health. J Dent Hyg Sci 17: 12-19, 2017. https://doi.org/10.17135/jdhs.2017.17.1.12

15. Choi YY: Relationship between asthma and dental caries in Korean adults: data from the Sixth Korea National Health and Nutrition Examination Survey. J Korean Soc Dent Hyg 19: 131-140, 2019. https://doi.org/10.13065/jksdh.20190010

16. Alavaikko S, Jaakkola MS, Tjäderhane L, Jaakkola JJ: Asthma and caries: a systematic review and meta-analysis. Am J Epidemiol 174: 631-641, 2011. https://doi.org/10.1093/aje/kwr129

17. Bozejac BV, Stojšin I, Đuric M, et al.: Impact of inhalation therapy on the incidence of carious lesions in patients with asthma and COPD. J Appl Oral Sci 25: 506-514, 2017. https://doi.org/10.1590/1678-7757-2016-0147

18. Ryberg M, Möller C, Ericson T: Effect of $\beta 2$-adrenoceptor agonists on saliva proteins and dental caries in asthmatic children. J Dent Res 66: 1404-1406, 1987. https://doi.org/10.1177/00220345870660082401

19. Friedrich N, Völzke H, Schwahn C, et al.: Inverse association between periodontitis and respiratory allergies. Clin Exp Allergy 36: 495-502, 2006. https://doi.org/10.1111/j.1365-2222.2006.02455.x

20. Lee KO, Lee KH, Kim DE: Long-term effects of syrup medication for asthma on the salivary invertase activity and deciduous caries. J Korean Acad Pediatr Dent 25: 421-429, 1998.

21. Kim DE: Influences of syrup medication for asthma on the plaque index and gingival index. J Korean Acad Pediatr Dent 25: 157-161, 1998. 